\title{
PATRONES REFERENCIALES DE PRODUCCIÓN LACRIMALEN CANINOS, EMPLEANDO LA PRUEBA DE SCHIRMER, EN UNA CLÍNICA DE LIMA METROPOLITANA
}

\author{
Reference Values of Lacrimal Production through the Schirmer Test in \\ Canines in a Veterinary Clinic in Lima City
}

\author{
Julia Moreno H. ${ }^{1}$, Víctor Fernández A. ${ }^{1}$, Ysaac Chipayo G. ${ }^{1}$ y Alberto Crespo P. ${ }^{1}$
}

\section{Resumen}

Se determinó la producción lacrimal normal en canes aparentemente sanos mediante la Prueba Lacrimal de Schirmer en la Clínica de Animales Menores de la Facultad de Medicina Veterinaria de la Universidad Nacional Mayor de San Marcos, Lima. Se evaluaron 123 perros agrupados según sexo, edad y tipo de cráneo. La producción lacrimal promedio fue $16.4 \pm 1.5 \mathrm{~mm} / \mathrm{min}$, con un rango de 11 a $20 \mathrm{~mm} / \mathrm{min}$, sin diferencia estadística debido a sexo o edad; sin embargo, la producción lacrimal de los canes braquicéfalos fue significativamente mayor $(17.0 \pm 1.2 \mathrm{~mm} / \mathrm{min}, \mathrm{p}<0.05)$ que los mesocéfalos $(16.4 \pm 1.7$ $\mathrm{mm} / \mathrm{min}$ ) y los dolicocéfalos $(15.6 \pm 1.1 \mathrm{~mm} / \mathrm{min})$.

Palabras clave: producción lacrimal, perros, prueba lacrimal de Schirmer

\section{Abstract}

The normal lacrimal production in apparently healthy dogs was determined by the Schirmer Tear in the Small Animals Clinic of the Faculty of Veterinary Medicine, Universidad Nacional Mayor de San Marcos, Lima. A total of 123 dogs grouped by sex, age, and skull type were evaluated. The mean lacrimal production was $16.4 \pm 1.5 \mathrm{~mm} / \mathrm{min}$, with a range of 11 to $20 \mathrm{~mm} / \mathrm{min}$, and without statistical difference due to sex and age; however, the lacrimal production of brachycephalic dogs was significantly higher $(17.0 \pm 1.2 \mathrm{~mm} /$ $\min , \mathrm{p}<0.05)$ that mesocephalic $(16.4 \pm 1.7 \mathrm{~mm} / \mathrm{min})$ and dolichocephalic dogs $(15.6 \pm 1.1$ $\mathrm{mm} / \mathrm{min})$.

Key words: lacrimal production, dogs, Schirmer tear test

\footnotetext{
${ }^{1}$ Clínica de Animales Menores, Facultad de Medicina Veterinaria, Universidad Nacional Mayor de San Marcos, Lima
} 
INTRODUCCIÓN

Las lágrimas son imprescindibles para mantener una córnea saludable. Una córnea en óptimas condiciones no tiene vasos sanguíneos; es decir, carece de irrigación, de allí que depende de las lágrimas para obtener el oxígeno, aminoácidos, vitamina $\mathrm{A}$, factores de crecimiento, nutrientes, sustancias antimicrobianas (lizosimas y leucocitos) e inhibidores de proteasas. Además, las lágrimas lubrican los párpados y permiten eliminar las partículas de material que se depositan en los ojos (Herrera, 1998; Carneiro, 2003; Vaz, 2003).

Las lágrimas resultan de una mezcla de secreciones que provienen de diversos tipos de glándulas, las cuales tienden a variar de acuerdo a las especies. Estas lágrimas forman una compleja película (película lacrimal precorneal) compuesta por varias capas que no se puedan reemplazar artificialmente (Severin, 1991).

La película lacrimal precorneal se compone de tres capas. La capa más externa (capa lipoide delgada) es secretada por las glándulas meibomianas, donde los lípidos retardan la evaporación y estabilizan la superficie refractaria para una visión precisa. La capa intermedia (capa acuosa) es producida por las glándulas lagrimales orbital y nictitante, y contiene más de 70 proteínas vitales para la salud de la superficie corneal. La capa más interna (mucina) es secretada por las células caliciformes conjuntivales y el epitelio corneal, y está compuesta por varios tipos de mucinas que permiten el anclaje de la película lacrimal a las microvellosidades de la superficie epitelial ocular (Herrera, 1998; Carneiro, 2003).

Una baja o nula secreción del componente acuoso de la película lacrimal precorneal tiende a causar cambios inflamatorios degenerativos en la córnea y la conjuntiva, denominados «Ojo Seco» o Queratoconjuntivitis Seca (QCS), la cual pue- de evolucionar en opacidad corneal y ceguera (Brooks, 1992; Kaswan y Bounous, 1995). Existen diferentes causas que pueden producir una disminución de la producción lacrimal; entre ellas, el virus del Distemper, toxicidad por sulfonamidas, uso tópico prolongado de atropina e injurias del nervio facial. Sin embargo, la etiología de la QCS no siempre puede ser determinada con exactitud. Existen diversos estudios serológicos e histopatológicos realizados en perros que revelan hallazgos similares a aquellos que se reportan en la QCS autoinmune humana (Herrera, 1998).

Se tiene evidencia que la medición de la producción de lágrimas es una prueba eficaz de diagnóstico para determinar el grado de deficiencia del sistema lacrimal. El sistema productor de lágrimas se evalúa de manera cualitativa a través del examen de la humedad y brillo de la superficie corneal y, de forma cuantitativa, mediante la Prueba Lacrimal de Schirmer (Brooks, 1992; Slatter, 2003). Convencionalmente se vienen aplicando otros dos métodos derivados y alternativos a la Prueba Lacrimal de Schirmer, como son la Prueba de Schirmer Modificada (con anestesia ocular) y la Prueba del Hilo Rojo de Fenol. En la clínica rutinaria se usa mayormente la Prueba Lacrimal de Schirmer (Saito y Kotani, 2001; Williams, 2005).

La prueba de Schirmer cuantifica, en milímetros, la humidificación del papel filtro en 60 segundos. En caninos normales, los valores del examen de Schirmer se extienden desde 15 hasta $25 \mathrm{~mm} / \mathrm{min}$; en tanto que valores que fluctúan entre 11 y $14 \mathrm{~mm} / \mathrm{min}$ corresponden a sospechoso o enfermedad subclínica y valores inferiores a $5 \mathrm{~mm} / \mathrm{min}$ a QCS grave (Gelatt, 1981, 2003). Asimismo, Severin (1991) considera como valores normales a aquellos superiores a $9 \mathrm{~mm} / \mathrm{min}$, sospechoso entre 5 y $8 \mathrm{~mm} / \mathrm{min}$ y QCS segura a valores inferiores a $5 \mathrm{~mm} / \mathrm{min}$. Por otro lado, Brooks (1992) considera que los valores normales varían entre 14 y $21.9 \mathrm{~mm} / \mathrm{min}$ y considera como sospechosos a valores entre $8 \mathrm{y}$ $10 \mathrm{~mm} / \mathrm{min}$. En general, valores menores de 
$10 \mathrm{~mm} / \mathrm{min}$ son considerados como deficiencia en la producción lacrimal (Laus, 1999). En un estudio realizado en Tokio, Japón, se encontró en perros beagles sanos valores de $18.9 \pm 2.6 \mathrm{~mm} / \mathrm{min}$ (Saito y Kotani, 2001).

Algunas variaciones en los valores de la prueba de Schirmer realizada en perros son causadas por fluctuaciones biológicas relacionadas a la edad y al sexo. En humanos, las condiciones medioambientales impactan en la producción lacrimal, en particular, las condiciones secas (áridas), que tienden a estar asociadas con disminución de la producción lacrimal (Rothschild et al., 2004).

El objetivo del presente estudio fue la determinación de valores de producción lagrimal que pueden servir de referencia en la zona de Lima en la evaluación de pacientes caninos con problemas oculares.

\section{Materiales y Métodos}

El estudio se llevó a cabo en la Clínica de Animales Menores de la Facultad de Medicina Veterinaria de la Universidad Nacional Mayor de San Marcos, Lima. Se utilizaron 123 animales que a la evaluación clínica resultaron aparentemente normales, libres de enfermedades sistémicas y de enfermedades oculares detectables. Los datos obtenidos fueron agrupados de acuerdo al sexo, tipo de cráneo (mesocéfalo, braquicéfalo y dolicocéfalo) y edad (0-3.9; 4-7.9; $\geq 8$ años) para diferenciar animales jóvenes, adultos y gerontes.

Se evaluó clínicamente la apariencia de los ojos y anexos (pestañas y párpados) para determinar la ausencia de anormalidades visibles, y en los animales aparentemente sanos se procedió a realizar la Prueba Lacrimal de Schirmer. Para esto, se empleó tiras de papel filtro Whatman N. ${ }^{\circ} 40$ (Ashless ${ }^{\circledR}$ ) de 5 $\mathrm{mm}$ de ancho por $40 \mathrm{~mm}$ de largo y con un doblez a $5 \mathrm{~mm}$ del extremo (Severin, 1991). Se expuso el saco conjuntival inferior y se le introdujo el doblez de la tira de papel filtro. Luego de 60 segundos de exposición se retiró la tira, se marcó la longitud alcanzada por la lágrima y se midió con una regla métrica simple la cantidad de papel humedecido desde el doblez en adelante. Las medidas se expresaron en milímetros por minuto. Luego se procedió de la misma forma con el otro ojo (Herrera, 1998).

El resultado por animal se obtuvo al sacar el promedio de ambos ojos y el resultado general se obtuvo determinando el promedio y la desviación estándar de los 123 animales. La relación entre la producción lacrimal y el sexo se empleó con la prueba de $\mathrm{T}$ Student de Independencia. La relación entre la producción lacrimal y el tipo de cráneo o la edad se evaluó con la prueba de Análisis de Varianza de una vía. Los datos fueron analizados con el paquete estadístico SPSS 13.0 - 2004.

\section{Resultados}

La distribución de los caninos por sexo correspondió a 60 hembras y 63 machos; por tipo de cráneo a 66 mesocéfalos, 37 braquicéfalos y 20 dolicocéfalos; y por edad en 62,39 y 22 canes de $0-3.9,4-7.9$ y $\geq 8$ años, respectivamente.

La producción lacrimal promedio de la población fue de $16.4 \pm 1.5 \mathrm{~mm} / \mathrm{min}$, sin que se haya encontrado diferencia estadística entre ojos (derecho: $16.5 \pm 1.6$; izquierdo: $16.4 \pm 1.5 \mathrm{~mm} / \mathrm{min}$ ) ni entre sexos o edades; sin embargo, se encontró una menor producción lacrimal para los braquicéfalos $(\mathrm{p}<0.05)$ aunque sin mayor importancia clínica (Cuadro 1). 


\section{Discusión}

El valor promedio de la producción lacrimal encontrado en el presente trabajo fue de $16.4 \pm 1.5 \mathrm{~mm} /$ minuto y todos los datos se distribuyeron dentro de un rango de 11 a 20 $\mathrm{mm} / \mathrm{min}$, lo cual concuerda con los valores de otros autores; así tenemos medidas de referencia de 10 a $25 \mathrm{~mm} / \mathrm{min}$ (Gelatt, 1981), de 14 a $21.9 \mathrm{~mm} / \mathrm{min}$ (Brooks, 1992), de 15 a $25 \mathrm{~mm} / \mathrm{min}$ (Laus, 1999), de 14 a $25 \mathrm{~mm} / \mathrm{min}$ (Saito y Kotani, 2001) y superiores a $9 \mathrm{~mm} /$ min (Severin, 1991).

El sexo no fue un factor de importancia en la producción lacrimal; sin embargo, el hallazgo de tres hembras ovariohisterectomizadas con valores de producción lacrimal bajos $(11,11.5$ y $12 \mathrm{~mm} / \mathrm{min})$ haría suponer la importancia de los esteroides sexuales en la secreción de la glándula lacrimal. Hay reportes que sostienen que, tanto la glándula lagrimal como la conjuntiva poseen receptores a los esteroides sexuales, por lo que en caso de hembras ovariohisterectomizadas, la producción de lágrimas disminuiría por la deficiencia de estrógenos (Peiffer y PetersonJones, 2002). Davidson y Kuonen (2004) mencionan que la deficiencia de estrógeno ha sido ligada al desarrollo de la QS así como la degeneración de la glándula lacrimal; de manera similar, en la mujer se observa cambios morfológicos adversos en la conjuntiva en proporción al tiempo de menopausia por deficiencia de estrógenos (Brincat, 2003).

La mayor producción lacrimal de los canes braquicéfalos puede deberse a la exoftalmia que presenta este tipo de cráneo (Sisson y Grossman, 2000), toda vez que ante una mayor exposición de la conjuntiva ocurre una mayor irritación, un mayor estímulo y por consiguiente un aumento en la producción lacrimal, aunque sin exceder los rangos de normalidad.

La producción lacrimal en humanos decrece notablemente a partir de los 60 años (Murube, 2002); sin embargo, esto no parece suceder en caninos ya que las medias de los tres grupos de edades (jóvenes, adultos y gerontes) fueron estadísticamente similares. Asimismo, no se encontró diferencias significativas en otros estudios.

\section{Conclusiones}

- El rango de la producción lacrimal de la población de caninos fue de $11 \mathrm{a} 20 \mathrm{~mm} /$ minuto y el promedio de $16.4 \pm 1.5 \mathrm{~mm} /$ minuto.

- No se encontraron diferencias estadísticas significativas en relación al sexo y la edad, pero los canes braquicéfalos presentaron una producción significativamente mayor que los canes mesocéfalos y dolicocéfalos.

\section{Literatura Citada}

1. Brooks D. 1992. Conceptos actuales de oftalmología veterinaria. Colegio Estadounidense de Oftalmología Veterinaria. [Internet], [04 junio 2005]. Disponible en: http://www.vetmed.ufl.edulSACS/ ophtho/04amveppa notesspanish.pdf

2. Brincat M. 2003. Piel y órganos de los sentidos en la menopausia. Departamento de Obstetricia y Ginecología del Hospital San Lucas de la Universidad de Malta. [Internet], [30 marzo 2006]. Disponible en: http://www.womanlab.com/ s p a n is h/professiona $1 \mathrm{~s} /$ menopausalIssues8.htm

3. Carneiro L. 2003. Aparelho lacrimal: Ceratoconjuntivite seca. Colegio Latinoamericano de Oftalmología Veterinaria. [Internet], [11 mayo 2005]. Disponible en: http://www.compuland.com.br/ aftalvet/cerato.htm

4. Davidson H, Kuonen V. 2004. The tear film and ocular mucins. Vet Ophthalmo 7(2): 71-77.

5. Gelatt K. 1981. Veterinary ophthalmology. London: Bailliere Tindall. 483 p. 
6. Gelatt K. 2003. Fundamentos de oftalmología veterinaria. London: Bailliere Tindall. $552 \mathrm{p}$.

7. Herrera D. 1998. Queratoconjuntivitis seca canina. Simposio ISVO/SOLOVE. Buenos Aires, Argentina. [Internet], [11 mayo 2005]. Disponible en: http://www. oftalmovet.com.ar

8. Kaswan L, Bounous D. 1995. Queratoconjuntivitis seca. Vet Med 90(6): 115-137.

9. Laus J. 1999. Doenças corneanas em pequenos animais. Rev Educ Cont CRMV-SP 2(1): 26-33.

10. Murube J. 2002. Ojo seco: anatomía y fisiología. Oftalmo Publicaciones [Internet], [04 mayo 2006]. [Online]. Disponible en: http://www.oftalmo.com/ publicaciones/ojoseco/indice.htm

11. Peiffer R, Peterson-Jones S. 2002. Oftalmología de pequeños animales. $3^{\text {ra }}$ ed. Madrid: Elsevier. 286 p.

12. Rothschild C, Sellon D, Bryan G, Gay J. 2004. Effects of trimethoprim- sulfadiazine on tear production and the fluctuations of Schirmer tear test values in horses. Vet Ophthalmol 7(6): 385-390.

13. Saito A, Kotani T. 2001. Estimation of lacrimal level and testing methods on normal beagles. Vet Ophthalmol 4(7): 7-11.

14. Severin G. 1991. Manual de oftalmología veterinaria. Buenos Aires, Argentina: Hemisferio Sur. 203 p.

15. Sisson S, Grossmam JD. 2000. Anatomía de los animales domésticos. $5^{\text {ta }}$ ed. Tomo II. Barcelona, España: Masson.

16. Slatter D. 2003. Fundamentos en oftalmología veterinaria. $3^{\text {ra }}$ ed. Buenos Aires, Argentina: Intermédica. 700 p.

17. Vaz O. 2003. Queratoconjuntivite seca. Hospital Veterinario do Porto. Portugal. [Internet], [28 julio 2005]. [Online]. Disponible en: http://www.hospvetporto.pt/

18. Williams D. 2005. Analysis of tear uptake by the Schirmer Tear test strip in the canine eye. Vet Ophthalmol 8(5): 325 330. 